Archive for

Organic Chemistry

Arkivoc 2018, part v, 0-0

\title{
Ring-closing metathesis as a key step to construct the 2,6-dihydropyrano[2,3- c]pyrazole ring system
}

\author{
Aurimas Bieliauskas, ${ }^{\mathrm{a}, \mathrm{b}}$ Sonata Krikštolaitytė, ${ }^{\mathrm{b}}$ Wolfgang Holzer, ${ }^{\mathrm{c}}$ and Algirdas Šačkus ${ }^{\mathrm{a}, \mathrm{b} *}$ \\ anstitute of Synthetic Chemistry, Kaunas University of Technology, K. Baršausko g. 59, Kaunas LT-51423, \\ Lithuania \\ ${ }^{b}$ Department of Organic Chemistry, Kaunas University of Technology, Radvileny pl. 19, Kaunas LT-50254, \\ Lithuania \\ c Department of Pharmaceutical Chemistry, University of Vienna, Althanstrasse 14, Viena 1090, Austria \\ Email: algirdas.sackus@ktu.lt
}

Received 11-25-2017

Accepted 02-17-2018

Published on line 07-07-2018

\section{Abstract}

A simple and efficient synthetic route to the 2,6-dihydropyrano[2,3-c]pyrazole ring system was developed by employing ring-closing metathesis (RCM) as a key step. The required diene substrate for the RCM reaction was prepared by a three-step procedure starting form 1-phenyl-1H-pyrazol-3-ol. Treatment of the obtained 4ethenyl-1-phenyl-3-[(prop-2-en-1-yl)oxy]-1H-pyrazole with Grubbs' first-generation catalyst afforded the target 2-phenyl-2,6-dihydropyrano[2,3-c]pyrazole. 2-(4-Fluorophenyl)- and 2-(4-bromophenyl)-2,6dihydropyrano[2,3-c]pyrazole were synthesized by an analogous way. The structures of the obtained heterocyclic products were unequivocally confirmed by detailed ${ }^{1} \mathrm{H},{ }^{13} \mathrm{C},{ }^{15} \mathrm{~N}$ and ${ }^{19} \mathrm{~F}$ NMR spectroscopic experiments and HRMS measurements. The optical properties of 2-phenyl-2,6-dihydropyrano[2,3-c]pyrazole were studied by UV-Vis and fluorescence spectroscopy.

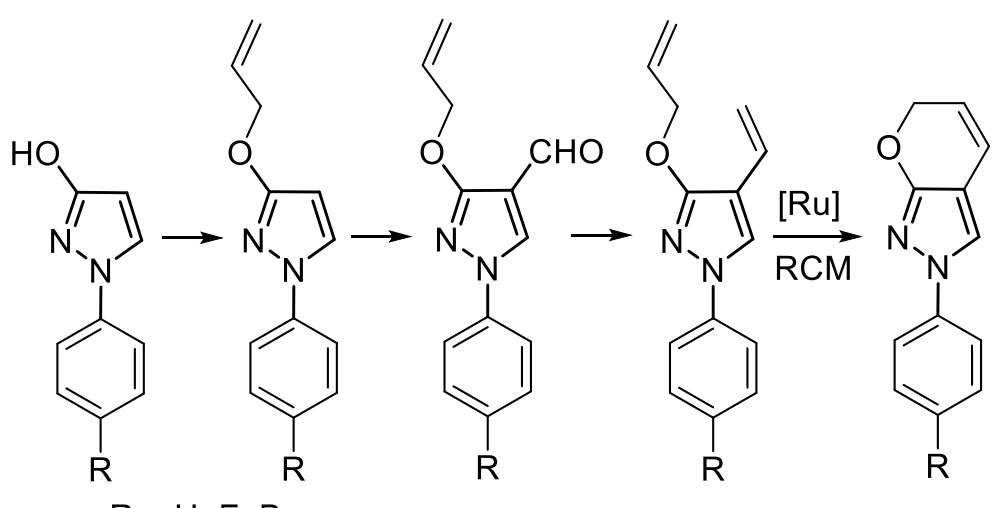

$\mathrm{R}=\mathrm{H}, \mathrm{F}, \mathrm{Br}$

Keywords: 1-Phenylpyrazol-3-ol, Wittig olefination, Ring-closing metathesis, 2,6-Dihydropyrano[2,3-c]pyrazole 


\section{Introduction}

The pyrano[2,3-c] pyrazole ring system (Fig. 1, A) is present in a wide variety of biologically active compounds. ${ }^{1}$ In recent years, there has been increasing interest in the chemistry of its dihydro analogues. The 1,4- and 2,4dihydropyrano[2,3-c]pyrazole ring systems, which correspond to tautomeric forms B and C (Fig. 1), often appear as the main structural motifs of anticancer, ${ }^{2-5}$ anti-inflammatory, ${ }^{6}$ and anti-diabetic agents. ${ }^{7}$ The known numerous methods for the preparation of these compounds are generally based on a multicomponent reaction of an aromatic aldehyde, 3-oxobutanoate, hydrazine hydrate, and malononitrile in the presence of a suitable catalyst. ${ }^{8}$ However, access to derivatives of 1,6- and 2,6-dihydropyrano[2,3-c]pyrazole isomers D and E (Fig. 1) is very limited, and their chemistry and biological properties remain largely unexplored. Derivatives of pyrano[2,3-c]pyrazol-6(1H)-one ${ }^{9}$ (Fig. 1, F) are structurally similar and are known as analgesic, antiinflammatory ${ }^{10}$ and antiviral agents. ${ }^{11}$

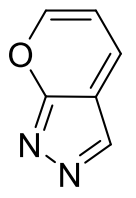

A

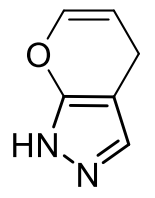

B pyrano[2,3-c]pyrazole 1,4-dihydropyrano[2,3-c]pyrazole<smiles>C1=Cc2cn[nH]c2OC1</smiles>

D

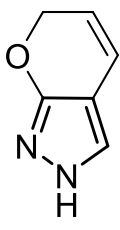

E

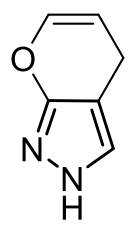

C

2,4-dihydropyrano[2,3-c]pyrazole

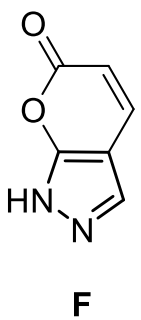

1,6-dihydropyrano[2,3-c]pyrazole 2,6-dihydropyrano[2,3-c]pyrazole

pyrano[2,3-c]pyrazol-6(1H)-one

Figure 1. Pyrano[2,3-c] pyrazole and its hydro derivatives.

In the present work, we describe a method for the construction of the 2,6-dihydropyrano[2,3-c]pyrazole ring system (Fig. 1, E) via a ring-closing metathesis (RCM) reaction. RCM reactions catalyzed by ruthenium alkylidene complexes ${ }^{12,13}$ have proven to be one of the most powerful tools for the construction of nonaromatic (hetero)carbocyclic compounds ${ }^{14-16}$, in particular, oxygen heterocycles. ${ }^{17-19}$ For example, treatment of 2-allyloxy-1-ethenylbenzene with Grubb's first-generation catalyst, $\left[\mathrm{Cl}_{2}\left(\mathrm{PCy}_{3}\right)_{2} \mathrm{Ru}=\mathrm{CHPh}{ }^{20}\right.$ afforded $2 \mathrm{H}$-1benzopyran in $95 \%$ yield. ${ }^{21}$

\section{Results and Discussion}

The synthetic strategy designed to construct the 2,6-dihydropyrano[2,3-c]pyrazole ring system employs a diene substrate that contains an ethene unit attached to an allyloxy unit onto the pyrazole core, which can participate in the RCM reaction (Scheme 1). As a starting material, we used 1-phenylpyrazol-3-ol (1a), which is readily accessible from the oxidation of 1-phenyl-3-pyrazolidinone. ${ }^{22-24}$ Recently, we applied this scaffold to 
obtain the novel pyrazolo[4,3-f][1,2,3]triazolo[5,1-c][1,4]oxazepine and pyrazolo[4',3':3,4]pyrido[1,2a]benzimidazole ring systems $\mathrm{s}^{25,26}$ and to prepare building blocks for the preparation of optoelectronic materials and fluorescent organic nanoparticles. ${ }^{27-30}$

The $O$-allylation of $\mathbf{1 a}$ with allyl bromide in the presence of $\mathrm{NaH}$ gave $O$-allylated pyrazole $2 \mathrm{a} .{ }^{31}$ To introduce a formyl group at the 4-position of the pyrazole ring, we employed a previously reported procedure based on the Vilsmeier-Haack reaction. ${ }^{32}$ Heating compound $2 \mathrm{a}$ with $\mathrm{POCl}_{3}$ in $\mathrm{N}, \mathrm{N}$-dimethylformamide (DMF) at $60{ }^{\circ} \mathrm{C}$ resulted in the formation of the desired pyrazole-4-carbaldehyde 3a in $91 \%$ yield (Scheme 1). The characteristic signals of aldehyde $3 a$ in the ${ }^{1} \mathrm{H}$ NMR spectrum were the singlets at $8.25(5-\mathrm{H})$ and $9.88 \mathrm{ppm}$ (CHO). The ${ }^{13} \mathrm{C}$ NMR spectrum contained the signal of a formyl carbon at $183.4 \mathrm{ppm}$.

Next, we investigated the conversion of aldehyde 3a into 4-ethenylpyrazole 4a. One of the most popular methods for the synthesis of alkenes from aldehydes and ketones is the Wittig reaction, which is based on the coupling of carbonyl compounds with single-substituted phosphonium ylides. ${ }^{33}$ To introduce a methylene group, methylenetriphenylphosphorane $\left(\mathrm{Ph}_{3} \mathrm{P}=\mathrm{CH}_{2} \leftrightarrow \mathrm{Ph}_{3} \mathrm{P}^{+}-\mathrm{CH}_{2}\right)$ generated by the addition of a base to a solution of methyltriphenylphosphonium bromide or iodide is typically used as an ylide source. ${ }^{34,35}$ For example, the Wittig reaction of benzaldehyde with methyltriphenylphosphonium iodide in the presence of $\mathrm{K}_{2} \mathrm{CO}_{3}$ in DME provided styrene in $90 \%$ yield. ${ }^{35}$ In our case, the reaction of aldehyde $3 a$ with methyltriphenylphosphonium iodide in the presence of $\mathrm{KOtBu}$ in toluene resulted in the formation of 4ethenylpyrazole $4 \mathrm{a}$ in $89 \%$ yield. The ${ }^{13} \mathrm{C}$ NMR spectrum of $4 a$ exhibited the corresponding signals of the newly formed vinyl carbon atoms at 113.4 and $125.1 \mathrm{ppm}$.

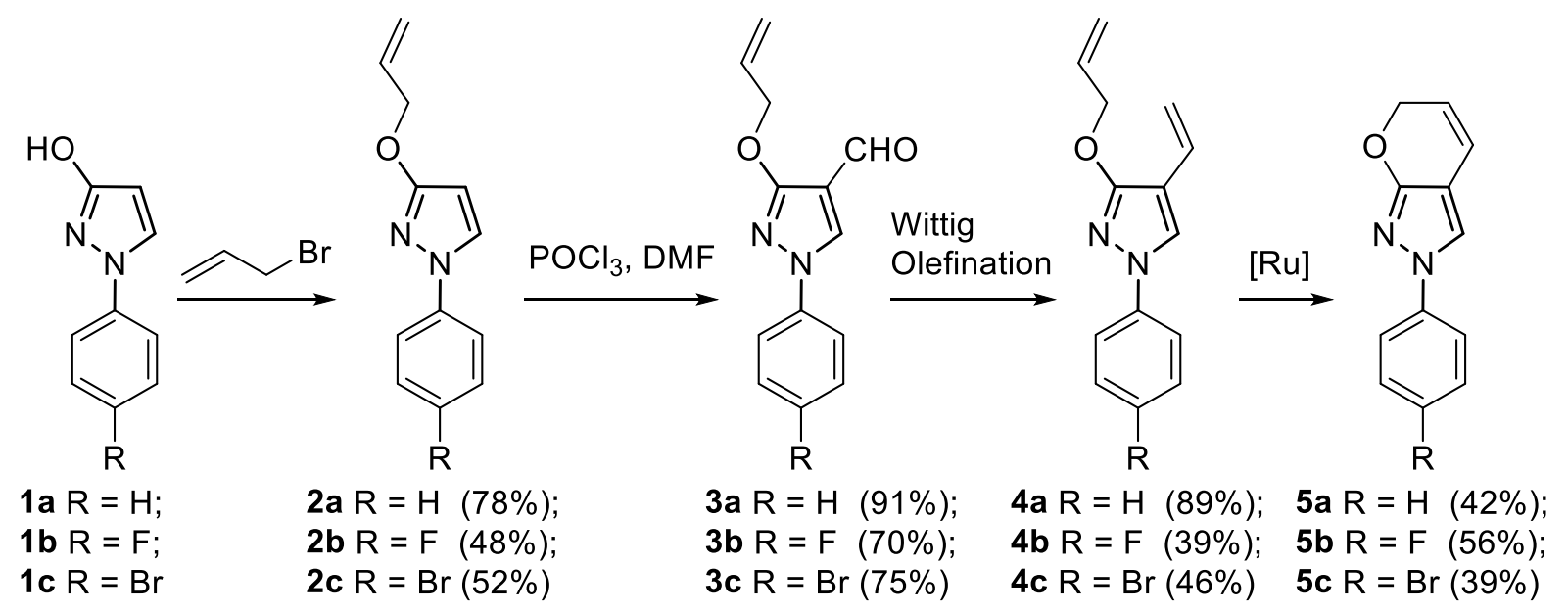

Scheme 1. Synthetic route to the 2,6-dihydropyrano[2,3-c]pyrazole ring system.

Having prepared the required diene $4 a$, we further investigated its RCM reaction in order to convert the latter into the target compound $\mathbf{5 a}$. When $\mathbf{4 a}$ was heated with Grubbs' first-generation catalyst in dichloromethane, no RCM reaction occurred. However, the replacement of the solvent with THF gave the desired 2-phenyl-2,6-dihydropyrano[2,3-c]pyrazole 5 a in $42 \%$ yield. The application of microwave heating allowed to shorten the RCM reaction time from $48 \mathrm{~h}$ to $3 \mathrm{~h}$, but the isolated yield of $5 \mathrm{a}$ was only $34 \%$.

The heterocyclic compounds of type 5 represent dihydropyrano[2,3-c]pyrazole substructures related to important functional organic molecules with wide biomedical applications. Because popular NMR prediction programs, such as $\mathrm{ACD} \mathrm{C}+\mathrm{H}$ predictor, ${ }^{36}$ depend on high-quality data with unambiguously assigned resonances, we carried out NMR studies with compound $\mathbf{5 a}$ in an attempt to fully map all the NMR signals for ${ }^{1} \mathrm{H},{ }^{13} \mathrm{C}$ and ${ }^{15} \mathrm{~N}$ as accurately as possible (Fig. 1). The desired results were achieved through a combination of 
standard NMR techniques, such as DEPT, gs-HSQC, gs-HMBC, COSY, TOCSY, NOESY ${ }^{37}, \mathrm{H}^{2 B C^{38}}$ and 1,1ADEQUATE ${ }^{39}$ experiments. The broad-band decoupled ${ }^{13} \mathrm{C}$ NMR spectrum of compound 5a showed resonances for 10 carbon atoms. The DEPT-90 and 135 spectra indicated the presence of 1 methylene and 6 methine carbon atoms. Comparison of the DEPT spectrum with the broad-band decoupled ${ }^{13} \mathrm{C} N \mathrm{NMR}$ spectrum revealed the presence of 3 quaternary carbons. The multiplicity-edited ${ }^{1} \mathrm{H}-{ }^{13} \mathrm{C} \mathrm{HSQC}$ spectrum indicated that the methylene protons $\mathrm{H}-6$ have one-bond connectivity with the C- 6 carbon at $68.8 \mathrm{ppm}$. Moreover, this also revealed heteronuclear interactions between the protons of two pairs of chemically equivalent methine groups (7.36-7.40 and 7.54-7.58 ppm), with their respective carbons, which resonated at 129.4 and 117.6, respectively. The data from the ${ }^{1} \mathrm{H}-{ }^{13} \mathrm{C} \mathrm{HMBC}$ spectrum revealed long-range correlations of the methylene protons with the quaternary carbon C-7a (at 161.9 ppm) and protonated carbons C-5 (at 119.0 ppm) and C-4 (118.1 ppm). The aforementioned protonated carbon C-4 showed correlation with quaternary carbon C-3a in the 1,1-ADEQUATE spectrum, which was also supported by the correlation of C-3 with C-3a. The ${ }^{15} \mathrm{~N} N M R$ data were obtained via a ${ }^{1} \mathrm{H}-{ }^{15} \mathrm{~N} \mathrm{HMBC}$ experiment. Both nitrogen atoms showed appropriate couplings to $\mathrm{H}-3$, and in the case of $\mathrm{N}-2$, it had strong coupling with the aromatic protons $2-\mathrm{H}$ and $6-\mathrm{H}$. The TOCSY spectrum showed that there were two distinct spin systems in the molecule. The ${ }^{1} \mathrm{H}-{ }^{1} \mathrm{H}$ connectivities within each spin system were confirmed using the data from the COSY, TOCSY and NOESY spectra.

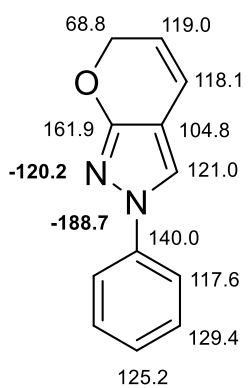

a)

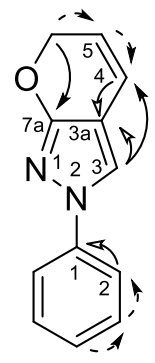

b)

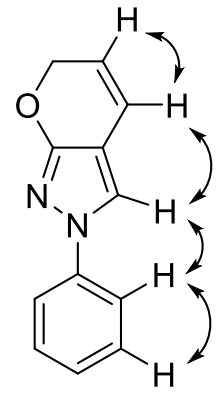

c)

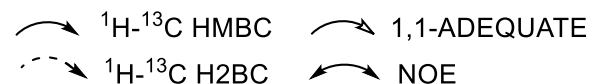

Figure 2. (a) ${ }^{13} \mathrm{C}$ and ${ }^{15} \mathrm{~N}$ (in bold) NMR chemical shifts of compound 5a. (b) Characteristic ${ }^{1} \mathrm{H}-{ }^{13} \mathrm{C} \mathrm{HMBC}, \mathrm{H} 2 \mathrm{BC}$ and 1,1-ADEQUATE correlations are represented by arrows. (c) Characteristic ${ }^{1} \mathrm{H}-{ }^{1} \mathrm{H}$ NOESY correlations are represented by arrows.

Compounds 5b,c were obtained analogously to compound 5a. Although the preparation of the starting compound $\mathbf{1 b}$ was reported in a patent applications, ${ }^{40,41}$ neither a detailed synthesis description nor spectroscopic data of the product were provided. The $O$-allylation of $\mathbf{1} \mathbf{b}$ and $\mathbf{1} \mathbf{c}^{42}$ and the subsequent formylation of $\mathbf{2} \mathbf{b}, \mathbf{c}$ produced aldehydes $\mathbf{3} \mathbf{b}, \mathbf{c}$ which after conversion to the corresponding ethenyl derivatives afforded the diene substrates $\mathbf{4 b}$ and $\mathbf{4 c}$. Treatment of $\mathbf{4 b , c}$ with Grubbs' first-generation catalyst resulted in the formation of the target compounds $\mathbf{5 b}$ and $\mathbf{5 c}$ in $56 \%$ and $39 \%$ yield, respectively.

The optical properties of compound 5a were investigated by UV-Vis spectroscopy and fluorometric measurements. The electronic absorption spectra of compound 5a in THF did not show absorption bands in the visible region of the electronic spectra, and it showed an absorption maximum at $310 \mathrm{~nm}$ (Fig. 3, a). Upon excitation of compound $\mathbf{5 a}$ at $320 \mathrm{~nm}$ (in THF solution), the fluorescence spectrum exhibited three peaks at 360, 381 and $395 \mathrm{~nm}$ (Fig. 3, b). The fluorescence quantum yield $\left(\Phi_{f}\right)$ of the solution was estimated by the integrating sphere method and gave a $\Phi_{\mathrm{f}}$ value of ca. $1 \%$. 


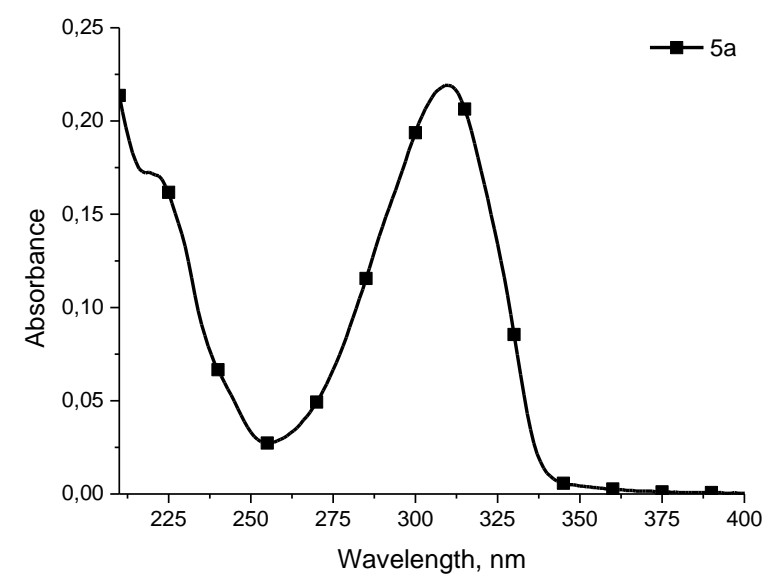

a)

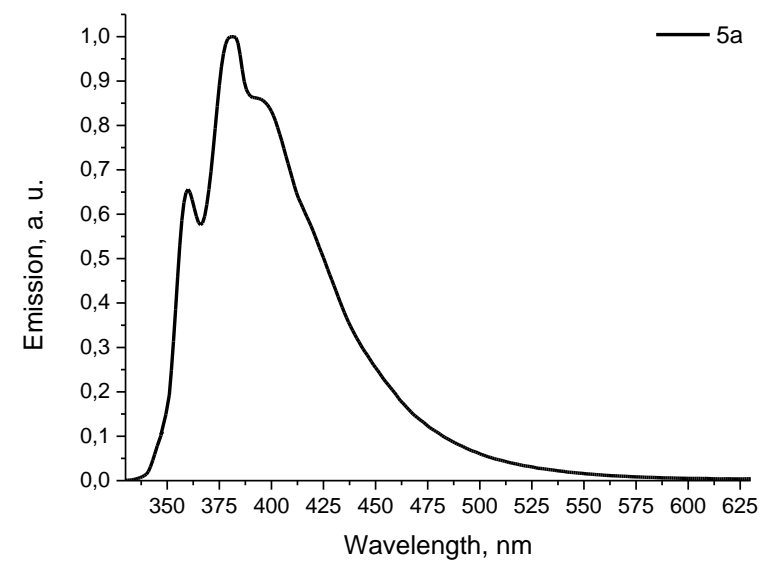

b)

Figure 3. (a) Absorption spectrum of 5 a in THF (0.1 mM, $298 \mathrm{~K})$; (b) fluorescence emission spectrum of $5 a$ in THF (? $\left.]_{\mathrm{ex}}=320 \mathrm{~nm}\right)$.

\section{Conclusions}

In summary, an efficient route to access the 2,6-dihydropyrano[2,3-c]pyrazole ring system was developed by employing ring-closing metathesis (RCM) as the key step. The structures of the synthesized 2-phenyl-, 2-(4bromophenyl)- and 2-(4-fluorophenyl)-2,6-dihydropyrano[2,3-c]pyrazoles were characterized by ${ }^{1} \mathrm{H},{ }^{13} \mathrm{C},{ }^{15} \mathrm{~N}$ and ${ }^{19} \mathrm{~F}$ NMR spectroscopy, UV-Vis and fluorescence spectroscopy and HRMS measurements.

\section{Experimental Section}

General. Microwave reactions were conducted using a CEM Discovery Synthesis Unit (CEM Corp., Matthews, $\mathrm{NC}$ ). The machine consists of a continuous focused microwave power delivery system with operator-selectable power output from 0 to $300 \mathrm{~W}$. The reactions were performed in glass vessels (capacity $10 \mathrm{~mL}$ ) sealed with septum. In the case of an open vessel conditions the reactions were performed in a round bottom flask (capacity $25 \mathrm{~mL}$ ) connected to a reflux condenser. All experiments were performed using a stirring option. For thin layer chromatography (TLC), Merck pre-coated TLC plates (Silica gel $60 \mathrm{~F}_{254}$ ) were employed. The purification of the products was performed using flash chromatography on a glass column with silica gel (high purity grade 9385 , pore size $60 \mathrm{~A}, 230-400$ mesh particle size). The melting points were determined in capillary tubes, on a capillary melting point apparatus Büchi Melting Point M-565 and are uncorrected. The ${ }^{1} \mathrm{H},{ }^{13} \mathrm{C}$ and ${ }^{15} \mathrm{~N}$ NMR spectra were recorded in $\mathrm{CDCl}_{3}$ solutions at $25^{\circ} \mathrm{C}$ on a Bruker Avance III $700\left(700 \mathrm{MHz}\right.$ for ${ }^{1} \mathrm{H}, 176$ $\mathrm{MHz}$ for ${ }^{13} \mathrm{C}, 71 \mathrm{MHz}$ for ${ }^{15} \mathrm{~N}$ ) spectrometer equipped with a $5 \mathrm{~mm} \mathrm{TCl}{ }^{1} \mathrm{H}-{ }^{13} \mathrm{C} /{ }^{15} \mathrm{~N} / \mathrm{D}$ z-gradient cryoprobe. The chemical shifts, expressed in ppm, were relative to tetramethylsilane (TMS). The ${ }^{15} \mathrm{~N}$ NMR spectra were referenced to neat, external nitromethane (coaxial capillary). ${ }^{19} \mathrm{~F} N \mathrm{NMR}$ spectra $(376.46 \mathrm{MHz}$, absolute referencing via $\delta$ ratio) were obtained on a Bruker Avance III 400 instrument with a 'directly' detecting broadband observe probe (BBO). The full and unambiguous assignments of the ${ }^{1} \mathrm{H},{ }^{13} \mathrm{C},{ }^{15} \mathrm{~N}$ and ${ }^{19} \mathrm{~F} N M R$ resonances were achieved using standard Bruker software and a combination of standard NMR spectroscopic techniques, such as DEPT, COSY, TOCSY, NOESY, gs-HSOC, gs-HMBC, H2BC and 1,1-ADEQUATE. The infrared 
spectra were recorded on a Bruker Vertex v70 FTIR spectrometer equipped with a diamond ATR accessory. The UV-vis spectra were recorded using $0.1 \mathrm{mM}$ solutions of the compounds in THF on a Shimadzu 2600 UV/Vis spectrometer. The fluorescence spectra were recorded on a FL920 fluorescence spectrometer from Edinburgh Instruments. The PL quantum yields were measured from dilute solutions by an absolute method using Edinburgh Instruments integrating sphere excited with a Xe lamp. Optical densities of the sample solutions were ensured to be below 0.1 to avoid reabsorption effects. All optical measurements were performed at rt under ambient conditions. HRMS spectra were recorded with a Bruker maXis or Bruker micrOTOF-QIII spectrometers.

1-(4-Fluorophenyl)-1H-pyrazol-3-ol (1b). An intensively stirred suspension of 4-fluorophenylhydrazine hydrochloride $(8.13 \mathrm{~g}, 50 \mathrm{mmol})$ in dry toluene/methanol $(1 / 1,100 \mathrm{~mL})$ was kept under inert atmosphere, and the potassium tert-butoxide $(16.83 \mathrm{~g}, 150 \mathrm{mmol})$ was added in one portion. The reaction mixture was stirred at room temperature for $30 \mathrm{~min}$. Then it was subsequently raised to $50^{\circ} \mathrm{C}$ and the ethyl acrylate $(150 \mathrm{mmol}$, $16.36 \mathrm{~mL}$ ) was added dropwise over the $15 \mathrm{~min}$. The reaction mixture was stirred at $50^{\circ} \mathrm{C}$ for $24 \mathrm{~h}$, diluted with $100 \mathrm{~mL}$ of water and the organic layer was separated. The aqueous phase was adjusted to a $\mathrm{pH}$ of 6 and extracted with ethyl acetate. The organic layers were combined, dried over $\mathrm{Na}_{2} \mathrm{SO}_{4}$, filtrated, and concentrated under reduced pressure. The residue was purified by column chromatography on silica gel with dichloromethane/methanol 100:3, v/v. To yield the 1-(4-fluorophenyl)pyrazolidin-3-one. Yield $6.31 \mathrm{~g}$ (70\%), white crystals, m.p. $109-11{ }^{\circ} \mathrm{C}$. IR (neat, $v_{\max } \mathrm{cm}^{-1}$ ): 3135, 2918, 1601, 1500, 1356, 1221, 1118. ${ }^{1} \mathrm{H}$ NMR (700 $\left.\mathrm{MHz}, \mathrm{CDCl}_{3}\right): \delta(\mathrm{ppm}) 2.56\left(\mathrm{t}, J 8.0 \mathrm{~Hz}, 2 \mathrm{H}, 4-\mathrm{CH}_{2}\right), 3.86\left(\mathrm{t}, J 8.0 \mathrm{~Hz}, 2 \mathrm{H}, 5-\mathrm{CH}_{2}\right), 7.00(\mathrm{~d}, J 6.4 \mathrm{~Hz}, 4 \mathrm{H}, \mathrm{Ph} 2,3,5,6-$ $\mathrm{H}), 9.14(\mathrm{~s}, 1 \mathrm{H}, \mathrm{NH}) .{ }^{13} \mathrm{C} \mathrm{NMR}\left(176 \mathrm{MHz}, \mathrm{CDCl}_{3}\right): \delta(\mathrm{ppm}) 29.9$ (C-4), 55.9 (C-5), 116.0 (d, $\left.{ }^{2} J_{\mathrm{C}, \mathrm{F}} 22.7 \mathrm{~Hz}, \mathrm{Ph} \mathrm{C}-3,5\right)$, $118.1\left(\mathrm{~d},{ }^{3} J_{\mathrm{C}, \mathrm{F}} 7.9 \mathrm{~Hz}\right.$, Ph C-2,6), 147.6 (d, $\left.{ }^{4} J_{\mathrm{C}, \mathrm{F}} 2.3 \mathrm{~Hz}, \mathrm{Ph} \mathrm{C}-1\right), 158.9$ (d, $\left.{ }^{1} J_{\mathrm{C}, \mathrm{F}} 241.6 \mathrm{~Hz}, \mathrm{Ph} \mathrm{C}-4\right), 175.7$ (C-3). ${ }^{15} \mathrm{~N}$ NMR (71 MHz, CDCl 3 ): $\delta$ (ppm) -278.3 (N-1), -230.2 (N-2). ${ }^{19} \mathrm{~F} \mathrm{NMR} \mathrm{(376} \mathrm{MHz}^{\mathrm{CDCl}} 3$ ): $\delta(\mathrm{ppm})-120.8$. HRMS (ESI TOF): $[\mathrm{M}+\mathrm{H}]^{+}$found 181.0771. $\left[\mathrm{C}_{9} \mathrm{H}_{10} \mathrm{FN}_{2} \mathrm{O}\right]^{+}$requires 181.0772.

The obtained 1-(4-fluorophenyl)pyrazolidin-3-one $(6.13 \mathrm{~g}, 34 \mathrm{mmol})$ and $\mathrm{FeCl}_{3} \cdot 6 \mathrm{H}_{2} \mathrm{O}(2.3 \mathrm{~g}, 8.5 \mathrm{mmol})$ were dissolved in dimethylformamide $(50 \mathrm{~mL})$. The reaction mixture was heated to $80{ }^{\circ} \mathrm{C}$, and gassed with oxygen for $2 \mathrm{~h}$. After gassing was stopped, the mixture was stirred for further $10 \mathrm{~h}$ maintaining at $80{ }^{\circ} \mathrm{C}$. Then it was poured into water $(100 \mathrm{~mL})$ and extracted with ethyl acetate $(3 \times 20 \mathrm{~mL})$. The organic layers were combined, washed with brine, dried over $\mathrm{Na}_{2} \mathrm{SO}_{4}$, filtrated, and concentrated under reduced pressure. The residue was purified by column chromatography on silica gel with hexane/ethylacetate $2: 1, \mathrm{v} / \mathrm{v}$, to yield the title compound 1b. Yield $4.91 \mathrm{~g}(81 \%)$, bright orange crystals, m.p. $152-154{ }^{\circ} \mathrm{C}$. IR (neat, $v_{\max } \mathrm{cm}^{-1}$ ): 3139,2938 , 1556, 1510, 1393, 1230, 1158. ${ }^{1} \mathrm{H}$ NMR $\left(700 \mathrm{MHz}, \mathrm{CDCl}_{3}\right): \delta(\mathrm{ppm}) 5.88(\mathrm{~d}, J 2.6 \mathrm{~Hz}, 1 \mathrm{H}, 4-\mathrm{H}), 7.11-7.18(\mathrm{~m}$, $2 \mathrm{H}, \mathrm{Ph} 3,5-\mathrm{H}), 7.43-7.48(\mathrm{~m}, 2 \mathrm{H}, \mathrm{Ph} 2,6-\mathrm{H}), 7.58(\mathrm{~d}, J 2.6 \mathrm{~Hz}, 1 \mathrm{H}, 5-\mathrm{H}) .{ }^{13} \mathrm{C} \mathrm{NMR}(176 \mathrm{MHz}, \mathrm{CDCl}): \delta(\mathrm{ppm}) 94.4$ (C-4), 116.6 (d, $\left.{ }^{2} J_{C, F} 23.0 \mathrm{~Hz}, \mathrm{Ph} \mathrm{C}-3,5\right), 120.8$ (d, $\left.{ }^{3} J_{C, F} 8.3 \mathrm{~Hz}, \mathrm{Ph} \mathrm{C}-2,6\right), 129.5$ (C-5), $136.0\left(\mathrm{~d},{ }^{4} J_{C, F} 2.8 \mathrm{~Hz}, \mathrm{Ph} \mathrm{C}-1\right)$, 160.9 (d, ${ }^{1} J_{C, F} 245.8 \mathrm{~Hz}$, Ph C-4), 164.1 (C-3). ${ }^{15} \mathrm{~N} \mathrm{NMR} \mathrm{(71} \mathrm{MHz,} \mathrm{CDCl} 3$ ): $\delta(p p m)-191.8(\mathrm{~N}-1),-135.2(\mathrm{~N}-2) .{ }^{19} \mathrm{~F}$ NMR (376 MHz, CDCl 3 ): $\delta(p p m)$-116.2. HRMS (ESI TOF): $[\mathrm{M}+\mathrm{H}]^{+}$found 179.0615. $\left[\mathrm{C}_{9} \mathrm{H}_{8} \mathrm{FN}_{2} \mathrm{O}\right]^{+}$requires 179.0615 .

General procedure for the allylation of $1 \mathrm{H}$-pyrazol-3-oles giving 3-[(prop-2-en-1-yl)oxy]-1H-pyrazoles (compounds $2 \mathrm{a}-\mathbf{c})$. The solution of $1 \mathrm{a}-\mathrm{c}(10 \mathrm{mmol})$ in dry DMF $(15 \mathrm{~mL})$ was cooled to $0^{\circ} \mathrm{C}$ under inert atmosphere and $\mathrm{NaH}$ (60\% dispersion in mineral oil, $400 \mathrm{mg}, 10 \mathrm{mmol}$ ) was added portionwise. After mixing for $15 \mathrm{~min}$ at $0^{\circ} \mathrm{C}$, the mixture was gradually warmed up to $40^{\circ} \mathrm{C}$ temperature and stirred for additional $15 \mathrm{~min}$. Then it was subsequently raised to $60^{\circ} \mathrm{C}$ and an allyl bromide $(12 \mathrm{mmol}, 1.0 \mathrm{~mL}$ ) was added dropwise over the $10 \mathrm{~min}$. The mixture was stirred at $60^{\circ} \mathrm{C}$ for $8 \mathrm{~h}$, diluted with $60 \mathrm{ml}$ of water and extracted with ethyl acetate. 
The organic layers were combined, washed with brine, dried over $\mathrm{Na}_{2} \mathrm{SO}_{4}$, filtrated, and concentrated under reduced pressure. The residue was purified by column chromatography on silica gel with hexane/ethylacetate 15:1, v/v. To yield compounds 2a-c.

1-Phenyl-3-[(prop-2-en-1-yl)oxy]-1H-pyrazole (2a). Yield $1.56 \mathrm{~g}(78 \%)$, yellow oil. IR (neat, $\mathrm{V}_{\mathrm{max}}, \mathrm{cm}^{-1}$ ): 3132 , 2927, 1541, 1505, 1396, 1236, 985, 936. ${ }^{1} \mathrm{H}$ NMR $\left(700 \mathrm{MHz}, \mathrm{CDCl}_{3}\right): \delta(\mathrm{ppm}) 4.79\left(\mathrm{dt}, J 5.6,1.4 \mathrm{~Hz}, 2 \mathrm{H}, \mathrm{O}-\mathrm{C}_{2}{ }^{-}\right.$ $\left.\mathrm{CH}=\mathrm{CH}_{2}\right), 5.26-5.32\left(\mathrm{~m}, 1 \mathrm{H}, \mathrm{O}-\mathrm{CH}_{2}-\mathrm{CH}=\underline{\mathrm{C}}_{2}\right), 5.42-5.48\left(\mathrm{~m}, 1 \mathrm{H}, \mathrm{O}-\mathrm{CH}_{2}-\mathrm{CH}=\mathrm{C}_{2}\right), 5.91(\mathrm{~d}, J 2.6 \mathrm{~Hz}, 1 \mathrm{H}, 4-\mathrm{H})$, $6.09-6.17\left(\mathrm{~m}, 1 \mathrm{H}, \mathrm{O}-\mathrm{CH}_{2}-\mathrm{C} \underline{H}=\mathrm{CH}_{2}\right), 7.19-7.22(\mathrm{~m}, 1 \mathrm{H}, \mathrm{Ph} 4-\mathrm{H}), 7.39-7.42(\mathrm{~m}, 2 \mathrm{H}, \mathrm{Ph} 3,5-\mathrm{H}), 7.59-7.62(\mathrm{~m}$, $2 \mathrm{H}, \mathrm{Ph} 2,6-\mathrm{H}), 7.73(\mathrm{~d}, J=2.6 \mathrm{~Hz}, 1 \mathrm{H}, 5-\mathrm{H}) .{ }^{13} \mathrm{C} \mathrm{NMR}\left(176 \mathrm{MHz}, \mathrm{CDCl}_{3}\right): \delta(\mathrm{ppm}) 70.0\left(\mathrm{O}-\mathrm{CH}_{2}-\mathrm{CH}=\mathrm{CH}_{2}\right), 94.1(\mathrm{C}-4)$, $117.9\left(\mathrm{O}-\mathrm{CH}_{2}-\mathrm{CH}=\underline{\mathrm{C}} \mathrm{H}_{2}\right), 118.0$ (Ph C-2,6), 125.4 (Ph C-4), 127.8 (C-5), 129.5 (Ph C-3,5), $133.4\left(\mathrm{O}-\mathrm{CH}_{2}-\underline{\mathrm{CH}}=\mathrm{CH}_{2}\right)$, 140.3 (Ph C-1), 164.3 (C-3). ${ }^{15} \mathrm{~N} \mathrm{NMR} \mathrm{(71} \mathrm{MHz,} \mathrm{CDCl} 3$ ): $\delta(p p m)-186.0(\mathrm{~N}-1),-119.3(\mathrm{~N}-2) .{ }^{30}$

1-(4-Fluorophenyl)-3-[(prop-2-en-1-yl)oxy]-1H-pyrazole (2b). Yield $1.05 \mathrm{~g}$ (48\%), bright yellow oil. IR (neat, $\left.V_{\max }, \mathrm{cm}^{-1}\right): 3098,2915,1547,1516,1392,1217,1175,991,942 .{ }^{1} \mathrm{H} \mathrm{NMR}\left(700 \mathrm{MHz}, \mathrm{CDCl}_{3}\right): \delta(\mathrm{ppm}) 4.77(\mathrm{dt}, J$ 5.6, $\left.1.3 \mathrm{~Hz}, 2 \mathrm{H}, \mathrm{O}-\mathrm{C}_{2}-\mathrm{CH}=\mathrm{CH}_{2}\right), 5.26-5.31\left(\mathrm{~m}, 1 \mathrm{H}, \mathrm{O}-\mathrm{CH}_{2}-\mathrm{CH}=\mathrm{C}_{2}\right), 5.41-5.48\left(\mathrm{~m}, 1 \mathrm{H}, \mathrm{O}-\mathrm{CH}_{2}-\mathrm{CH}=\underline{C}_{2}\right), 5.90$ $(\mathrm{d}, J 2.6 \mathrm{~Hz}, 1 \mathrm{H}, 4-\mathrm{H}), 6.07-6.15\left(\mathrm{~m}, 1 \mathrm{H}, \mathrm{O}-\mathrm{CH}_{2}-\underline{\mathrm{H}}=\mathrm{CH}_{2}\right), 7.07-7.11(\mathrm{~m}, 2 \mathrm{H}, \mathrm{Ph} 3,5-\mathrm{H}), 7.52-7.56(\mathrm{~m}, 2 \mathrm{H}, \mathrm{Ph}$ 2,6-H), 7.65 (d, J $2.6 \mathrm{~Hz}, 1 \mathrm{H}, 5-\mathrm{H}) .{ }^{13} \mathrm{C} \mathrm{NMR}\left(176 \mathrm{MHz}, \mathrm{CDCl}_{3}\right): 69.9\left(\mathrm{O}-\mathrm{CH}_{2}-\mathrm{CH}=\mathrm{CH}_{2}\right), 94.0(\mathrm{C}-4), 116.1\left(\mathrm{~d},{ }^{2} \mathrm{~J}_{\mathrm{C}, \mathrm{F}}\right.$ $23.0 \mathrm{~Hz}, \mathrm{Ph} \mathrm{C}-3,5), 118.0\left(\mathrm{O}-\mathrm{CH}_{2}-\mathrm{CH}=\underline{C H}_{2}\right), 119.6$ (d, ${ }^{3} \mathrm{~J}, \mathrm{~F}$ 8.2 Hz, Ph C-2,6), 127.9 (C-5), $133.3\left(\mathrm{O}-\mathrm{CH}_{2}-\underline{\mathrm{C}} \mathrm{H}=\mathrm{CH}_{2}\right)$, $136.6\left(\mathrm{~d},{ }^{4} J_{C, F} 2.7 \mathrm{~Hz}\right.$, Ph C-1), 160.5 (d, ${ }^{1} J_{C, F} 244.5 \mathrm{~Hz}$, Ph C-4), $164.3(\mathrm{C}-3) .{ }^{15} \mathrm{~N} \mathrm{NMR}(71 \mathrm{MHz}, \mathrm{CDCl} 3): \delta(\mathrm{ppm})-$ $187.8(\mathrm{~N}-1),-118.7$ (N-2). HRMS (ESI TOF): $[\mathrm{M}+\mathrm{Na}]^{+}$found 241.0748. [ $\left.\mathrm{C}_{12} \mathrm{H}_{11} \mathrm{FN}_{2} \mathrm{NaO}\right]^{+}$requires 241.0748.

1-(4-Bromophenyl) 3-[(prop-2-en-1-yl)oxy]-1H-pyrazole (2c). Yield $1.45 \mathrm{~g}(52 \%)$, colorless oil. IR (neat, $V_{\max }$ $\mathrm{cm}^{-1}$ ): 3154, 2916, 1547, 1498, 1385, 1236, 1179, 982, 932. ${ }^{1} \mathrm{H}$ NMR (700 MHz, CDCl $): \delta(p p m) 4.77(\mathrm{dt}, J 5.6$, $\left.1.3 \mathrm{~Hz}, 2 \mathrm{H}, \mathrm{O}-\underline{\mathrm{C}}_{2}-\mathrm{CH}=\mathrm{CH}_{2}\right), 5.26-5.31\left(\mathrm{~m}, 1 \mathrm{H}, \mathrm{O}-\mathrm{CH}_{2}-\mathrm{CH}=\underline{C}_{2}\right), 5.42-5.46\left(\mathrm{~m}, \mathrm{O}-\mathrm{CH}_{2}-\mathrm{CH}=\mathrm{C}_{2}\right), 5.92(\mathrm{~d}, J 2.6$ $\mathrm{Hz}, 1 \mathrm{H}, 4-\mathrm{H}), 6.06-6.15\left(\mathrm{~m}, 1 \mathrm{H}, \mathrm{O}-\mathrm{CH}_{2}-\underline{\mathrm{C}}=\mathrm{CH}_{2}\right), 7.47-7.53(\mathrm{~m}, 4 \mathrm{H}, \mathrm{Ph}$ 2,3,5,6-H), $7.70(\mathrm{~d}, J 2.6 \mathrm{~Hz}, 1 \mathrm{H}, 5-\mathrm{H})$. ${ }^{13} \mathrm{C}$ NMR (176 MHz, CDCl 3$): \delta(p p m) 70.0\left(\mathrm{O}-\underline{C H}_{2}-\mathrm{CH}=\mathrm{CH}_{2}\right), 94.7(\mathrm{C}-4), 118.1\left(\mathrm{O}-\mathrm{CH}_{2}-\mathrm{CH}=\underline{C H}_{2}\right), 118.3(\mathrm{Ph} \mathrm{C}-4)$, 119.3 and 132.4 (Ph C-2,3,5,6), 127.8 (C-5), $133.3\left(\mathrm{O}-\mathrm{CH}_{2}-\underline{\mathrm{C}} \mathrm{H}=\mathrm{CH}_{2}\right), 139.3$ (Ph C-1), 164.4 (C-3). ${ }^{15} \mathrm{~N} \mathrm{NMR} \mathrm{(71}$ $\left.\mathrm{MHz}, \mathrm{CDCl}_{3}\right): \delta(\mathrm{ppm})-188.1(\mathrm{~N}-1), \mathrm{N}-2$ was not found. HRMS (ESI TOF): $[\mathrm{M}+\mathrm{Na}]^{+}$found 300.9947 $\left[\mathrm{C}_{12} \mathrm{H}_{11}{ }^{79} \mathrm{BrN}_{2} \mathrm{NaO}\right]^{+}$requires 300.9947 .

General procedure for the formylation of 3-[(prop-2-en-1-yl)oxy]-1H-pyrazoles giving 3-[(prop-2-en-1yl)oxy]-1H-pyrazole-4-carbaldehydes (compounds 3a-c). Phosphorus oxychloride (1.86 mL, $20 \mathrm{mmol}$ ) was added dropwise to dry DMF $(1.55 \mathrm{~mL}, 20 \mathrm{mmol})$ at $-10^{\circ} \mathrm{C}$ and the resulting mixture was stirred at the same temperature for 10-20 min until the Vilsmeier-Haack complex formed. Then, the appropriate 3-[(prop-2-en-1yl)oxy]-1H-pyrazole 2a-c $(5 \mathrm{mmol})$ in DMF (15 mL) was added to the Vilsmeier-Haack complex and the temperature was slowly raised to $70^{\circ} \mathrm{C}$ and maintained for $12 \mathrm{~h}$. The reaction mixture was cooled in an ice bath, cautiously quenched with ice-cold water $(100 \mathrm{~mL})$, and basified with $10 \% \mathrm{NaHCO}_{3}$ solution. The precipitate was filtered off. The filtrate was extracted with ethyl acetate which was washed with brine, dried over anhydrous $\mathrm{Na}_{2} \mathrm{SO}_{4}$, filtrated and then concentrated. The residue was purified by column chromatography on silica gel with hexane/ethylacetate $3: 1, \mathrm{v} / \mathrm{v}$. To yield compounds $3 a-c$.

1-Phenyl-3-[(prop-2-en-1-yl)oxy]-1H-pyrazole-4-carbaldehyde (3a). Yield $1.04 \mathrm{~g}$ (91\%), bright yellow crystals, m.p. $107-109^{\circ} \mathrm{C}$. IR (neat, $v_{\max }, \mathrm{cm}^{-1}$ ): 3126, 2938, 1665, 1552, 1501, 1391, 1222, 1205, 993, 943. ${ }^{1} \mathrm{H}$ NMR (700 $\left.\mathrm{MHz}, \mathrm{CDCl}_{3}\right): \delta(\mathrm{ppm}) 4.92\left(\mathrm{dt}, J 5.7,1.3 \mathrm{~Hz}, 2 \mathrm{H}, \mathrm{O}-\mathrm{C}_{2}-\mathrm{CH}=\mathrm{CH}_{2}\right), 5.30-5.35\left(\mathrm{~m}, 1 \mathrm{H}, \mathrm{O}-\mathrm{CH}_{2}-\mathrm{CH}=\mathrm{C}_{2}\right), 5.45-$ $5.50\left(\mathrm{~m}, 1 \mathrm{H}, \mathrm{O}-\mathrm{CH}_{2}-\mathrm{CH}=\underline{\mathrm{C}}_{2}\right), 6.11-6.18\left(\mathrm{~m}, 1 \mathrm{H}, \mathrm{O}-\mathrm{CH}_{2}-\mathrm{C}_{\underline{H}}=\mathrm{CH}_{2}\right), 7.32(\mathrm{t}, J 7.4 \mathrm{~Hz}, 1 \mathrm{H}, \mathrm{Ph} 4-\mathrm{H}), 7.44-7.49(\mathrm{~m}$, $2 \mathrm{H}, \mathrm{Ph} 3,5-\mathrm{H}), 7.62-7.66(\mathrm{~m}, 2 \mathrm{H}, \mathrm{Ph} 2,6-\mathrm{H}), 8.25(\mathrm{~s}, 1 \mathrm{H}, 5-\mathrm{H}), 9.88(\mathrm{~s}, 1 \mathrm{H}, \mathrm{C} \underline{\mathrm{HO}}) .{ }^{13} \mathrm{C} \mathrm{NMR}\left(176 \mathrm{MHz}^{\left.\mathrm{C} C \mathrm{Cl}_{3}\right): \delta}\right.$ (ppm) $70.2\left(\mathrm{O}-\underline{\mathrm{C}} \mathrm{H}_{2}-\mathrm{CH}=\mathrm{CH}_{2}\right), 111.6(\mathrm{C}-4), 118.7\left(\mathrm{O}-\mathrm{CH}_{2}-\mathrm{CH}=\underline{\mathrm{C}} \mathrm{H}_{2}\right), 118.9$ (Ph C-2,6), 127.4 (Ph C-4), 129.5 (C-5), 
129.7 (Ph C-3,5), $132.6\left(\mathrm{O}-\mathrm{CH}_{2}-\underline{\mathrm{C}} \mathrm{H}=\mathrm{CH}_{2}\right), 139.1$ (Ph C-1), 163.6 (C-3), 183.4 (므O). ${ }^{15} \mathrm{~N} \mathrm{NMR}(71 \mathrm{MHz}, \mathrm{CDCl} 3): \delta$ (ppm) -179.2 (N-1), -118.0 (N-2). HRMS (ESI TOF): [M+Na] found 229.0974. [ $\left.\mathrm{C}_{13} \mathrm{H}_{13} \mathrm{~N}_{2} \mathrm{O}_{2}\right]^{+}$requires 229.0972. 1-(4-Fluorophenyl)-3-[(prop-2-en-1-yl)oxy]-1H-pyrazole-4-carbaldehyde (3b). Yield $0.86 \mathrm{~g}$ (70\%), bright brown crystals, m.p. $134-136{ }^{\circ} \mathrm{C}$. IR (neat, $v_{\max } \mathrm{cm}^{-1}$ ): 3126, 2946, 1669, 1564, 1502, 1390, 1224, 1209, 989, $941 .{ }^{1} \mathrm{H}$ NMR (700 MHz, CDCl 3$): \delta(p p m) 4.89\left(\mathrm{dt}, J 5.7,1.3 \mathrm{~Hz}, 2 \mathrm{H}, \mathrm{O}-\mathrm{C}_{2}-\mathrm{CH}=\mathrm{CH}_{2}\right), 5.30-5.34\left(\mathrm{~m}, 1 \mathrm{H}, \mathrm{O}-\mathrm{CH}_{2}-\mathrm{CH}=\mathrm{C}_{2}\right)$, $5.44-5.48\left(\mathrm{~m}, 1 \mathrm{H}, \mathrm{O}-\mathrm{CH}_{2}-\mathrm{CH}=\underline{\mathrm{CH}}_{2}\right), 6.09-6.16\left(\mathrm{~m}, 1 \mathrm{H}, \mathrm{O}-\mathrm{CH}_{2}-\mathrm{C}_{\mathrm{H}}=\mathrm{CH}_{2}\right), 7.12-7.17(\mathrm{~m}, 2 \mathrm{H}, \mathrm{Ph} 3,5-\mathrm{H}), 7.57-$ $7.64(\mathrm{~m}, 2 \mathrm{H}, \mathrm{Ph} 2,6-\mathrm{H}), 8.19(\mathrm{~s}, 1 \mathrm{H}, 5-\mathrm{H}), 9.86(\mathrm{~s}, 1 \mathrm{H}, \mathrm{C} \underline{\mathrm{HO}}) .{ }^{13} \mathrm{C} \mathrm{NMR}\left(176 \mathrm{MHz}, \mathrm{CDCl}_{3}\right): \delta(\mathrm{ppm}) 70.2\left(\mathrm{O}-\underline{\mathrm{C}} \mathrm{H}_{2^{-}}\right.$ $\left.\mathrm{CH}=\mathrm{CH}_{2}\right), 111.6(\mathrm{C}-4), 116.6$ (d, $\left.{ }^{2} J_{\mathrm{C}, \mathrm{F}} 23.2 \mathrm{~Hz}, \mathrm{Ph} \mathrm{C}-3,5\right), 118.8\left(\mathrm{O}-\mathrm{CH}_{2}-\mathrm{CH}=\underline{C H}_{2}\right), 120.7\left(\mathrm{~d},{ }^{3} \mathrm{C}_{\mathrm{C}, \mathrm{F}} 8.4 \mathrm{~Hz}, \mathrm{Ph} \mathrm{C}-2,6\right)$, 129.6 (C-5), $132.5\left(\mathrm{O}-\mathrm{CH}_{2}-\underline{\mathrm{C}} \mathrm{H}=\mathrm{CH}_{2}\right), 135.4$ (d, $\left.{ }^{4} \mathrm{~J}_{\mathrm{C}, \mathrm{F}} 2.8 \mathrm{~Hz}, \mathrm{Ph} \mathrm{C}-1\right), 161.6$ (d, $\left.{ }^{1} \mathrm{~J}_{\mathrm{C}, \mathrm{F}} 247.4 \mathrm{~Hz}, \mathrm{Ph} \mathrm{C}-4\right), 163.6$ (C-3), 183.4 (ㅁO). $\left.{ }^{15} \mathrm{~N} \mathrm{NMR} \mathrm{(71} \mathrm{MHz}, \mathrm{CDCl}_{3}\right): \delta$ (ppm) -181.2 (N-1), -117.3 (N-2). HRMS (ESI TOF): [M+Na] ${ }^{+}$found 269.0696. [ $\left.\mathrm{C}_{13} \mathrm{H}_{11} \mathrm{FN}_{2} \mathrm{NaO}_{2}\right]^{+}$requires 269.0697.

1-(4-Bromophenyl)-3-[(prop-2-en-1-yl)oxy]-1H-pyrazole-4-carbaldehyde (3c). Yield $1.15 \mathrm{~g}$ (75\%), bright brown crystals, m.p. $125-127^{\circ} \mathrm{C}$. IR (neat, $v_{\max } \mathrm{cm}^{-1}$ ): $3124,2923,1665,1554,1496,1418,1221,1207,988$, 939. ${ }^{1} \mathrm{H}$ NMR $\left(700 \mathrm{MHz}, \mathrm{CDCl}_{3}\right): \delta(\mathrm{ppm}) 4.90\left(\mathrm{dt}, J 5.8,1.2 \mathrm{~Hz}, 2 \mathrm{H}, \mathrm{O}-\mathrm{C}_{2}-\mathrm{CH}=\mathrm{CH}_{2}\right), 5.31-5.35\left(\mathrm{~m}, 1 \mathrm{H}, \mathrm{O}-\mathrm{CH}_{2}-\right.$ $\left.\mathrm{CH}=\underline{\mathrm{C}}_{2}\right), 5.45-5.50\left(\mathrm{~m}, 1 \mathrm{H}, \mathrm{O}-\mathrm{CH}_{2}-\mathrm{CH}=\mathrm{C}_{2}\right), 6.10-6.17\left(\mathrm{~m}, 1 \mathrm{H}, \mathrm{O}-\mathrm{CH}_{2}-\mathrm{C}_{\underline{H}}=\mathrm{CH}_{2}\right), 7.50-7.66(\mathrm{~m}, 4 \mathrm{H}, \mathrm{Ph}$ 2,3,5,6-H), $8.23(\mathrm{~s}, 1 \mathrm{H}, 5-\mathrm{H}), 9.87(\mathrm{~s}, 1 \mathrm{H}, \mathrm{C} \underline{\mathrm{HO}}) .{ }^{13} \mathrm{C} \mathrm{NMR}\left(176 \mathrm{MHz}, \mathrm{CDCl}_{3}\right): \delta(\mathrm{ppm}) 70.3\left(\mathrm{O}-\mathrm{CH}_{2}-\mathrm{CH}_{=} \mathrm{CH}_{2}\right), 111.9$ (C-4), $118.9\left(\mathrm{O}-\mathrm{CH}_{2}-\mathrm{CH}=\underline{C H}_{2}\right), 120.3$ and 132.8 (Ph C-2,3,5,6), 120.7 (Ph C-4), 129.5 (C-5), $132.4\left(\mathrm{O}-\mathrm{CH}_{2-}\right.$ $\underline{\mathrm{CH}}=\mathrm{CH}_{2}$ ), 138.1 (Ph C-1), $163.6(\mathrm{C}-3), 183.4(\underline{\mathrm{C}} \mathrm{HO}) .{ }^{15} \mathrm{~N} \mathrm{NMR}\left(71 \mathrm{MHz}, \mathrm{CDCl}_{3}\right): \delta(\mathrm{ppm})-181.5(\mathrm{~N}-1),-118.3(\mathrm{~N}-$ 2). HRMS (ESI TOF): [M+Na] ${ }^{+}$found 328.9897. $\left[\mathrm{C}_{13} \mathrm{H}_{11}{ }^{79} \mathrm{BrN}_{2} \mathrm{NaO}_{2}\right]^{+}$requires 328.9896.

General procedure for the Wittig olefination of 3-[(prop-2-en-1-yl)oxy]-1H-pyrazole-4-carbaldehydes giving 4-ethenyl-3-[(prop-2-en-1-yl)oxy]-1H-pyrazoles (compounds 4a-c). To a suspension of methyltriphenylphosphonium iodide $(1.83 \mathrm{~g}, 4.5 \mathrm{mmol})$ in dry toluene $(60 \mathrm{~mL})$ under inert atmosphere, the potassium tertbutoxide $(1.01 \mathrm{~g}, 9 \mathrm{mmol})$ was added in one portion. The reaction mixture was stirred at room temperature for $30 \mathrm{~min}$ and subsequently refluxed for another $30 \mathrm{~min}$. Formation of the ylide can be visibly observed by its persistent yellow color. After refluxing, the reaction mixture was allowed to come to room temperature and kept in an ice bath, followed by dropwise addition of an appropriate aldehyde 3a-c (3 mmol) dissolved in dry toluene $(30 \mathrm{~mL})$. Then the reaction was carried out in room temperature for 3-5 hours, and the progress was monitored by TLC. Upon completion, the reaction was quenched by saturated solution of ammonium chloride and the organic layer was extracted with ethyl acetate several times. The organic layers were combined, dried over $\mathrm{Na}_{2} \mathrm{SO}_{4}$, filtrated, and concentrated under reduced pressure. The residue was purified by column chromatography on silica gel with hexane/ethylacetate $15: 1, \mathrm{v} / \mathrm{v}$ to yield compounds $4 \mathrm{a}-\mathrm{c}$.

4-Ethenyl-1-phenyl-3-[(prop-2-en-1-yl)oxy]-1H-pyrazole (4a). Yield $0.60 \mathrm{~g}(89 \%)$, colorless oil. IR (neat, $V_{\text {max }}$ $\left.\mathrm{cm}^{-1}\right): 3081,2928,1564,1500,1396,1235,1205,989,940 .{ }^{1} \mathrm{H} \mathrm{NMR}\left(700 \mathrm{MHz}, \mathrm{CDCl}_{3}\right): \delta(\mathrm{ppm}) 4.89$ (dt, J 5.5, $\left.1.5 \mathrm{~Hz}, 2 \mathrm{H}, \mathrm{O}-\underline{\mathrm{C}}_{2}-\mathrm{CH}=\mathrm{CH}_{2}\right), 5.18\left(\mathrm{dd}, \mathrm{J} 11.3,1.7 \mathrm{~Hz}, 1 \mathrm{H}, \mathrm{Pyr} 4-\mathrm{CH}=\mathrm{C}_{2}\right), 5.28-5.31\left(\mathrm{~m}, 1 \mathrm{H}, \mathrm{O}-\mathrm{CH}_{2}-\mathrm{CH}=\mathrm{C} \underline{H}_{2}\right)$, $5.45-5.50\left(\mathrm{~m}, 1 \mathrm{H}, \mathrm{O}-\mathrm{CH}_{2}-\mathrm{CH}=\underline{\mathrm{C}}_{2}\right), 5.74\left(\mathrm{dd}, \mathrm{J} 17.7,1.7 \mathrm{~Hz}, 1 \mathrm{H}, \mathrm{Pyr} 4-\mathrm{CH}=\underline{\mathrm{C}}_{2}\right), 6.12-6.20\left(\mathrm{~m}, 1 \mathrm{H}, \mathrm{O}-\mathrm{CH}_{2}-\right.$ $\left.\mathrm{C} \underline{\mathrm{H}}=\mathrm{CH}_{2}\right), 6.55\left(\mathrm{dd}, J 17.7,11.3 \mathrm{~Hz}, 1 \mathrm{H}, \mathrm{Pyr} 4-\underline{\mathrm{H}}=\mathrm{CH}_{2}\right), 7.18-7.22(\mathrm{~m}, 1 \mathrm{H}, \mathrm{Ph} 4-\mathrm{H}), 7.39-7.43(\mathrm{~m}, 2 \mathrm{H}, \mathrm{Ph} 3,5-$ $\mathrm{H}), 7.57-7.61(\mathrm{~m}, 2 \mathrm{H}, \mathrm{Ph} 2,6-\mathrm{H}), 7.75(\mathrm{~s}, 1 \mathrm{H}, 5-\mathrm{H}) .{ }^{13} \mathrm{C} \mathrm{NMR}\left(176 \mathrm{MHz}, \mathrm{CDCl}_{3}\right): \delta(\mathrm{ppm}) 69.7\left(\mathrm{O}-\mathrm{CH}_{2}-\mathrm{CH}=\mathrm{CH}_{2}\right)$, 108.7 (C-4), $113.4\left(\mathrm{Pyr} 4-\mathrm{CH}=\underline{\mathrm{C}} \mathrm{H}_{2}\right), 117.6\left(\mathrm{O}-\mathrm{CH}_{2}-\mathrm{CH}=\underline{C H}_{2}\right), 117.7(\mathrm{Ph} \mathrm{C}-2,6), 125.1$ (Pyr 4- $\left.\underline{\mathrm{CH}}=\mathrm{CH}_{2}\right), 125.2$ (C-5), 125.3 (Ph C-4), 129.5 (Ph C-3,5), $133.4\left(\mathrm{O}-\mathrm{CH}_{2}-\mathrm{CH}=\mathrm{CH}_{2}\right), 140.0$ (Ph C-1), 161.7 (C-3). ${ }^{15} \mathrm{~N} \mathrm{NMR}\left(71 \mathrm{MHz}, \mathrm{CDCl}_{3}\right)$ : $\delta(\mathrm{ppm})-189.4(\mathrm{~N}-1),-119.6(\mathrm{~N}-2)$. HRMS (ESI TOF): $[\mathrm{M}+\mathrm{Na}]^{+}$found 249.1000. $\left[\mathrm{C}_{14} \mathrm{H}_{14} \mathrm{~N}_{2} \mathrm{NaO}\right]^{+}$requires 249.0998.

4-ethenyl-1-(4-fluorophenyl)-3-[(prop-2-en-1-yl)oxy]-1H-pyrazole (4b). Yield $0.29 \mathrm{~g}$ (39\%), colorless oil. IR (neat, $\left.v_{\max }, \mathrm{cm}^{-1}\right): 3086,2929,1564,1511,1396,1226,1205,989,941 .{ }^{1} \mathrm{H} \mathrm{NMR}\left(700 \mathrm{MHz}, \mathrm{CDCl}_{3}\right): \delta$ (ppm) 4.86 
(dt, J 5.4, $\left.1.4 \mathrm{~Hz}, 2 \mathrm{H}, \mathrm{O}-\mathrm{C}_{2}-\mathrm{CH}=\mathrm{CH}_{2}\right), 5.17$ (dd, J 11.3, $\left.1.7 \mathrm{~Hz}, 1 \mathrm{H}, \mathrm{Pyr} 4-\mathrm{CH}=\underline{\mathrm{C}}_{2}\right), 5.27-5.31\left(\mathrm{~m}, 1 \mathrm{H}, \mathrm{O}-\mathrm{CH}_{2}-\right.$ $\left.\mathrm{CH}=\underline{\mathrm{C}}_{2}\right), 5.43-5.49\left(\mathrm{~m}, 1 \mathrm{H}, \mathrm{O}-\mathrm{CH}_{2}-\mathrm{CH}=\underline{\mathrm{C}}_{2}\right), 5.72\left(\mathrm{dd}, J 17.7,1.7 \mathrm{~Hz}, 1 \mathrm{H}, \mathrm{Pyr} 4-\mathrm{CH}=\mathrm{C}_{2}\right), 6.11-6.18(\mathrm{~m}, 1 \mathrm{H}, \mathrm{O}-$ $\mathrm{CH}_{2}-\mathrm{C} \underline{H}=\mathrm{CH}_{2}$ ), 6.52 (dd, J 17.7, $\left.11.3 \mathrm{~Hz}, 1 \mathrm{H}, \mathrm{Pyr} 4-\mathrm{C} \underline{H}=\mathrm{CH}_{2}\right), 7.07-7.12(\mathrm{~m}, 2 \mathrm{H}, \mathrm{Ph} 3,5-\mathrm{H}), 7.51-7.55(\mathrm{~m}, 2 \mathrm{H}, \mathrm{Ph}$ 2,6-H), 7.66 (s, $1 \mathrm{H}, 5-\mathrm{H}) .{ }^{13} \mathrm{C} \mathrm{NMR}\left(176 \mathrm{MHz}, \mathrm{CDCl}_{3}\right): \delta(\mathrm{ppm}) 69.7$ (O- $\left.\mathrm{CH}_{2}-\mathrm{CH}=\mathrm{CH}_{2}\right), 108.8$ (C-4), 113.5 (Pyr 4$\left.\mathrm{CH}=\underline{C H}_{2}\right), 116.2\left(\mathrm{~d},{ }^{2} J_{\mathrm{C}, \mathrm{F}} 22.9 \mathrm{~Hz}, \mathrm{Ph} \mathrm{C}-3,5\right), 117.6\left(\mathrm{O}-\mathrm{CH}_{2}-\mathrm{CH}=\underline{C H}_{2}\right), 119.4\left(\mathrm{~d},{ }^{3} J_{\mathrm{C}, \mathrm{F}} 8.1 \mathrm{~Hz}, \mathrm{Ph} \mathrm{C}-2,6\right), 125.0$ (Pyr 4$\left.\underline{\mathrm{CH}}=\mathrm{CH}_{2}\right), 125.3(\mathrm{C}-5), 133.4\left(\mathrm{O}-\mathrm{CH}_{2}-\underline{\mathrm{CH}}=\mathrm{CH}_{2}\right), 136.4$ (d, $\left.{ }^{4} J_{\mathrm{C}, \mathrm{F}} 2.6 \mathrm{~Hz}, \mathrm{Ph} \mathrm{C}-1\right), 160.5$ (d, $\left.{ }^{1} J_{\mathrm{C}, \mathrm{F}} 244.5 \mathrm{~Hz}, \mathrm{Ph} \mathrm{C}-4\right)$, $161.8(\mathrm{C}-3) .{ }^{15} \mathrm{~N}$ NMR $\left(71 \mathrm{MHz}, \mathrm{CDCl}_{3}\right): \delta$ (ppm) -191.2 (N-1), -119.0 (N-2). HRMS (ESI TOF): [M+Na] ${ }^{+}$found 267.0906. $\left[\mathrm{C}_{14} \mathrm{H}_{13} \mathrm{FN}_{2} \mathrm{NaO}\right]^{+}$requires 267.0904.

1-(4-Bromophenyl)-4-ethenyl-3-[(prop-2-en-1-yl)oxy]-1H-pyrazole (4c). Yield $0.42 \mathrm{~g}$ (46\%), colorless oil. IR (neat, $\left.v_{\max }, \mathrm{cm}^{-1}\right): 3083,2925,1563,1493,1392,1231,1181,989,937 .{ }^{1} \mathrm{H} \mathrm{NMR}\left(700 \mathrm{MHz}, \mathrm{CDCl}_{3}\right): \delta$ (ppm) 4.86 (dt, J 5.5, $\left.1.4 \mathrm{~Hz}, 2 \mathrm{H}, \mathrm{O}-\mathrm{C}_{2}-\mathrm{CH}=\mathrm{CH}_{2}\right), 5.18$ (dd, J 11.3, $\left.1.7 \mathrm{~Hz}, 1 \mathrm{H}, \mathrm{Pyr} 4-\mathrm{CH}=\mathrm{C}_{2}\right), 5.27-5.31\left(\mathrm{~m}, 1 \mathrm{H}, \mathrm{O}-\mathrm{CH}_{2}-\right.$ $\left.\mathrm{CH}=\underline{\mathrm{C}}_{2}\right), 5.43-5.48\left(\mathrm{~m}, 1 \mathrm{H}, \mathrm{O}-\mathrm{CH}_{2}-\mathrm{CH}=\underline{\mathrm{C}}_{2}\right), 5.73\left(\mathrm{dd}, \mathrm{J} 17.7,1.7 \mathrm{~Hz}, 1 \mathrm{H}, \mathrm{Pyr} 4-\mathrm{CH}=\mathrm{C}_{2}\right), 6.10-6.17(\mathrm{~m}, 1 \mathrm{H}, \mathrm{O}-$ $\mathrm{CH}_{2}-\mathrm{C} \underline{H}=\mathrm{CH}_{2}$ ), 6.51 (dd, J 17.7, $\left.11.3 \mathrm{~Hz}, 1 \mathrm{H}, \mathrm{Pyr} 4-\mathrm{C} \underline{H}=\mathrm{CH}_{2}\right), 7.43-7.53(\mathrm{~m}, 4 \mathrm{H}, \mathrm{Ph}$ 2,3,5,6-H), $7.71(\mathrm{~s}, 1 \mathrm{H}, 5-\mathrm{H})$. ${ }^{13} \mathrm{C} \mathrm{NMR} \mathrm{(176} \mathrm{MHz,} \mathrm{CDCl} 3$ ): $\delta$ (ppm) $69.8\left(\mathrm{O}-\underline{\mathrm{CH}}_{2}-\mathrm{CH}=\mathrm{CH}_{2}\right), 109.3$ (C-4), 113.9 (Pyr 4-CH= $\left.\underline{C H}_{2}\right), 117.7\left(\mathrm{O}-\mathrm{CH}_{2}-\right.$ $\left.\mathrm{CH}=\underline{\mathrm{CH}}_{2}\right), 118.2$ (Ph C-4), 119.0 and 132.4 (Ph C-2,3,5,6), 124.9 (Pyr 4- $\left.\underline{\mathrm{CH}}=\mathrm{CH}_{2}\right), 125.1$ (C-5), $133.3\left(\mathrm{O}-\mathrm{CH}_{2-}\right.$ $\underline{\mathrm{C}} \mathrm{H}=\mathrm{CH}_{2}$ ), 139.1 (Ph C-1), $161.9(\mathrm{C}-3) .{ }^{15} \mathrm{~N} \mathrm{NMR}$ (71 MHz, $\left.\mathrm{CDCl}_{3}\right): \delta$ (ppm) -191.5 (N-1), -119.9 (N-2). HRMS (ESI TOF): $[\mathrm{M}+\mathrm{Na}]^{+}$found 327.0104. $\left[\mathrm{C}_{14} \mathrm{H}_{13}{ }^{79} \mathrm{BrN}_{2} \mathrm{NaO}\right]^{+}$requires 327.0103 .

\section{General procedure for the ring-closing metathesis of 4-ethenyl-3-[(prop-2-en-1-yl)oxy]-1H-pyrazoles giving 2,6-dihydropyrano[2,3-c]pyrazoles (compounds 5a-c)}

Method A. Bis(tricyclohexylphosphine)benzylidene ruthenium dichloride $(41 \mathrm{mg}, 0.05 \mathrm{mmol}$ ) was added to a solution of an appropriate diene $4 a-c(1.0 \mathrm{mmol})$ in dry degassed tetrahydrofuran $(10 \mathrm{~mL})$. The reaction mixture was refluxed under argon atmosphere for $24 \mathrm{~h}$. Since the reaction was incomplete, and the catalyst had become deactivated, another portion of it $(41 \mathrm{mg}, 0.05 \mathrm{mmol}$ ) was added (total catalyst loading $10 \mathrm{~mol}$ $\%)$. The reaction mixture was refluxed for another $24 \mathrm{~h}$. Subsequently the solvent was removed under vacuum and the residue was purified by column chromatography on silica gel with hexane/ethylacetate $6: 1, \mathrm{v} / \mathrm{v}$. The yields of compounds 5 a (83 mg, 42\%), 5b (121 mg, 56\%) and 5c (108 mg, 39\%).

Method B. Bis(tricyclohexylphosphine)benzylidene ruthenium dichloride $(41 \mathrm{mg}, 0.05 \mathrm{mmol}$ ) was added to a solution of diene $4 \mathbf{a}(99 \mathrm{mg}, 0.5 \mathrm{mmol})$ in dry degassed tetrahydrofuran $(5 \mathrm{~mL})$. The reaction mixture was heated in a microwave reactor $(150 \mathrm{~W})$ to $65^{\circ} \mathrm{C}$ for $3 \mathrm{~h}$. Standard workup and purification according to the Method A yielded compound $\mathbf{5 a}$ (23 $\mathrm{mg}, 23 \%)$.

Method C. Bis(tricyclohexylphosphine)benzylidene ruthenium dichloride ( $82 \mathrm{mg}, 0.1 \mathrm{mmol}$ ) was added to a solution of diene $4 \mathrm{a}(198 \mathrm{mg}, 1.0 \mathrm{mmol})$ in dry degassed tetrahydrofuran $(10 \mathrm{~mL})$. The reaction mixture was refluxed in a microwave reactor $(150 \mathrm{~W})$ for $3 \mathrm{~h}$, under open vessel conditions with an inert gas sparging. Glass capillary for Ar purging were immersed through the reflux condenser into the solution maintaining the inert gas introduction. The solvent was kept at the constant volume. Standard workup and purification according to the Method A yielded compound 5 a (67 mg, 34\%).

2-Phenyl-2,6-dihydropyrano[2,3-c]pyrazole (5a). Pale crystals, m.p. 88-90 ${ }^{\circ} \mathrm{C}$. IR (neat, $v_{\max }, \mathrm{cm}^{-1}$ ): 3116,2927 , 1581, 1502, 1406, 1202. ${ }^{1} \mathrm{H}$ NMR (700 MHz, CDCl $): \delta(p p m) 5.03(\mathrm{dd}, J 3.3,2.0 \mathrm{~Hz}, 2 \mathrm{H}, 6-\mathrm{H}), 5.56$ (dt, J 9.8, 3.4 $\mathrm{Hz}, 1 \mathrm{H}, 5-\mathrm{H}), 6.47(\mathrm{dt}, J$ 9.8, $1.9 \mathrm{~Hz}, 1 \mathrm{H}, 4-\mathrm{H}), 7.18(\mathrm{t}, J 7.4 \mathrm{~Hz}, 1 \mathrm{H}, \mathrm{Ph} 4-\mathrm{H}), 7.37-7.41(\mathrm{~m}, 2 \mathrm{H}, \mathrm{Ph} \mathrm{3,5-H),} \mathrm{7.53} \mathrm{(s,}$

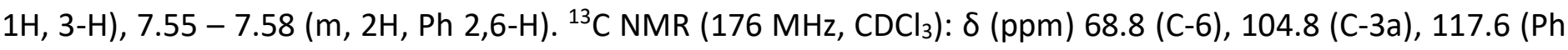
C-2,6), 118.2 (C-4), 119.1 (C-5), 121.1 (C-3), 125.3 (Ph C-4), 129.4 (Ph C-3,5), 140.1 (Ph C-1), 161.9 (C-7a). ${ }^{15} \mathrm{~N}$ NMR (71 MHz, CDCl 3 ): $\delta(p p m)$-188.7 (N-2), -120.2 (N-1). HRMS (ESI TOF): [M+H] $]^{+}$found 199.0864. $\left[\mathrm{C}_{12} \mathrm{H}_{11} \mathrm{~N}_{2} \mathrm{O}\right]^{+}$requires 199.0866 . 
2-(4-Fluorophenyl)-2,6-dihydropyrano[2,3-c]pyrazole (5b). Pale crystals, m.p. $104-107{ }^{\circ} \mathrm{C}$. IR (neat, $\mathrm{V}_{\max }, \mathrm{cm}^{-}$ $\left.{ }^{1}\right): 3133,2927,1590,1510,1399,1206 .{ }^{1} \mathrm{H}$ NMR $\left(700 \mathrm{MHz}, \mathrm{CDCl}_{3}\right): \delta(\mathrm{ppm}) 5.01(\mathrm{dd}, J 3.2,1.7 \mathrm{~Hz}, 2 \mathrm{H}, 6-\mathrm{H})$, 5.54 (dt, J 9.8, 3.4 Hz, 1H, 5-H), 6.44 (dt, J 9.8, $1.5 \mathrm{~Hz}, 1 \mathrm{H}, 4-\mathrm{H}), 7.04-7.09(\mathrm{~m}, 2 \mathrm{H}, \mathrm{Ph} \mathrm{3,5-H),} \mathrm{7.44} \mathrm{(s,} \mathrm{1H,} \mathrm{3-H),}$ $7.48-7.52(\mathrm{~m}, 2 \mathrm{H}, \mathrm{Ph} 2,6-\mathrm{H}) .{ }^{13} \mathrm{C}$ NMR (176 MHz, CDCl 3$): \delta(\mathrm{ppm}) 68.8(\mathrm{C}-6), 104.9$ (C-3a), 116.2 (d, ${ }^{2} \mathrm{~J}_{\mathrm{C}, \mathrm{F}} 22.9$ Hz, Ph C-3,5), 118.0 (C-4), 119.1 (C-5), 119.3 (d, ${ }^{3} J_{C, F} 8.2$ Hz, Ph C-2,6), $121.2(C-3), 136.4$ (d, ${ }^{4} J_{C, F} 2.6$ Hz, Ph C-1), $160.4\left(\mathrm{~d},{ }^{1} J_{\mathrm{C}, \mathrm{F}} 244.4 \mathrm{~Hz}\right.$, Ph C-4), 161.9 (C-7a). ${ }^{15} \mathrm{~N} \mathrm{NMR}\left(71 \mathrm{MHz}, \mathrm{CDCl}_{3}\right): \delta(\mathrm{ppm})-190.6(\mathrm{~N}-2),-119.7(\mathrm{~N}-1) .{ }^{19} \mathrm{~F}$ NMR (376 MHz, CDCl 3 ): $\delta(p p m)$-117.7. HRMS (ESI TOF): $[\mathrm{M}+\mathrm{Na}]^{+}$found 239.0591. $\left[\mathrm{C}_{12} \mathrm{H}_{9} \mathrm{FN}_{2} \mathrm{NaO}^{+}\right.$requires 239.0591.

2-(4-Bromophenyl)-2,6-dihydropyrano[2,3-c]pyrazole (5c). White crystals, m.p. $133-135^{\circ} \mathrm{C}$. IR (neat, $\mathrm{v}_{\max } \mathrm{cm}$ $\left.{ }^{1}\right): 3134,2927,1592,1495,1398,1207 .{ }^{1} \mathrm{H}$ NMR $\left(700 \mathrm{MHz}, \mathrm{CDCl}_{3}\right): \delta(\mathrm{ppm}) 5.03(\mathrm{dd}, J 3.3,2.0 \mathrm{~Hz}, 2 \mathrm{H}, 6-\mathrm{H})$, $5.57(\mathrm{dt}, J$ 9.8, $3.4 \mathrm{~Hz}, 1 \mathrm{H}, 5-\mathrm{H}), 6.45(\mathrm{dt}, J$ 9.8, $1.9 \mathrm{~Hz}, 1 \mathrm{H}, 4-\mathrm{H}), 7.42-7.50(\mathrm{~m}, 5 \mathrm{H}, 3-\mathrm{H}$ and $\mathrm{Ph}-2,3,5,6) .{ }^{13} \mathrm{C}$ NMR (176 MHz, CDCl 3 ): $\delta$ (ppm) 68.9 (C-6), 105.3 (C-3a), 117.9 (C-4), 118.1 (Ph C-4), 119.0 and 132.4 (Ph C2,3,5,6), 119.6 (C-5), 120.9 (C-3), 139.1 (Ph C-1), 162.0 (C-7a). ${ }^{15} \mathrm{~N} \mathrm{NMR} \mathrm{(71} \mathrm{MHz,} \mathrm{CDCl} 3$ ): $\delta$ (ppm) -190.8 (N-2), 120.5 (N-1). HRMS (ESI TOF): $[\mathrm{M}+\mathrm{H}]^{+}$found 276.9973. $\left[\mathrm{C}_{12} \mathrm{H}_{10}{ }^{79} \mathrm{BrN}_{2} \mathrm{O}\right]^{+}$requires 276.9971.

\section{Supplementary Material}

NMR spectra $\left({ }^{1} \mathrm{H},{ }^{13} \mathrm{C}\right.$, DEPT-135 ${ }^{13} \mathrm{C},{ }^{1} \mathrm{H}-{ }^{15} \mathrm{~N} \mathrm{HMBC},{ }^{1} \mathrm{H}-{ }^{13} \mathrm{C}$, gs-HSQC, and $60 \mathrm{~Hz}$ 1,1- ADEQUATE) of compound $\mathbf{5 a}$ are available in the Supplementary material file.

\section{References}

1. Das, D.; Banerjee, R.; Mitra, A. J. Chem. Pharm. Res. 2014, 6, 108-116.

2. Salama, S. K.; Mohamed, M. F.; Darweesh, A. F.; Elwahy, A. H. M.; Abdelhamid, I. A. Bioorg. Chem. 2017, 71, 19-29.

https://doi.org/10.1016/j.bioorg.2017.01.009

3. Yan, C.; Theodorescu, D.; Miller, B.; Kumar, V.; Ross, D.; Wempe, M. F. Bioorg. Med. Chem. Lett. 2016, 26, 5815-5818.

https://doi.org/10.1016/j.bmcl.2016.10.021

4. Kastoh, H.; Muhammad, M. T.; Khan, J. J. A.; Rashed, S.; Khan, A.; Perven, S.; Javaid, K.; Atia-tul-Wanab, Khan, K. Bioorg. Chem. 2016, 65, 61-72.

5. Adibi, H.; Hosseinzadeh, L.; Farhadi, S.; Ahmadi, F. J. Rep. Pharm. Sci. 2013, 2, 27-35.

6. Zaki, M. E. A. ; Soliman, H. A. ; Hiekal, O. A. ; Rashad, A. E. Z. Naturforsch. C 2006, 61 c, 1-5. https://doi.org/10.1515/znc-2006-1-201

7. Qvortrup, K.; Jensen, J. F.; Sørensen, M. S.; Kouskoumvekaki, I.; Petersen, R. K.; Taboureau, O.; Kristiansen, K.; Nielsen, T. E. PLoS ONE 2017, 12(2): e0162642.

https://doi.org/10.1371/journal.pone.0162642

8. Myrboh, B.; Mecadon, H.; Rohman, M. R.; Rajbangshi, M.; Kharkongor, I.; Laloo, B. M.; Kharbangar, I.;

Ksiar, B. Org. Prep. Proc. Int. 2013, 45, 253-303. https://doi.org/10.1080/00304948.2013.798566

9. Khan, M. A.; Cosenza, A. G.; Ellis, G. P. J. Heterocycl. Chem. 1982, 19, 1077-1085. 
10. Kuo, S.-C.; Huang, L. -J.; Nakammura, H. J. Med Chem. 1984, 27, 539-544. https://doi.org/10.1021/jm00370a020

11. Shamroukh, A. H.; Zaki, M. E. A.; Morsy, E. M. H.; Abdel-Motti, F. M.; Abdel-Megeid, F. M. E. Archiv. Pharm. 2007, 340, 236-243.

https://doi.org/10.1002/ardp.200700005

12. Grubbs, R. H.; Miller, S. J.; Fu, G. C. Acc. Chem. Res. 1995, 28, 446-452. https://doi.org/10.1021/ar00059a002

13. Deiters, A.; Martin, S. F. Chem. Rev. 2004, 104, 2199-2238.

https://doi.org/10.1021/cr0200872

14. Chattopadhyay, S. K.; Karmakar, S.; Biswas, T.; Majumdar, K. C.; Rahaman, H.; Roy, B. Tetrahedron, 2007, 63, 3919-3952.

https://doi.org/10.1016/i.tet.2007.01.063

15. Undheim, K. Amino Acids 2008, 34, 357-402.

https://doi.org/10.1007/s00726-007-0512-5

16. Krikstolaityte, S.; Sackus, A.; Romming, C.; Undheim, K. Tetrahedron: Asymmetry 2011, 12, 393-398. https://doi.org/10.1016/S0957-4166(01)00049-0

17. Jacques, R.; Pal, R.; Parker, N. A.; Sear, C. E.; Smith, P. W.; Ribaucourt, A.; Hodgson, D. M. Org. Biomol. Chem. 2016, 14, 5875-5893.

https://doi.org/10.1039/C6OB00593D

18. van Otterlo, W. A. L.; Ngidi, E. L.; Coyanis, E. M.; de Konig, C. B. Tetrahedron Lett. 2003, 44, 311-313. https://doi.org/10.1016/S0040-4039(02)02522-4

19. Dammacco, M.; Degennaro, L.; Florio, S.; Luisi, R.; Musio, B.; Altomare, A. J. Org. Chem. 2009, 74, 63196322.

https://doi.org/10.1021/jo9011943

20. Vougioukalakis, G. C.; Grubbs, R. H. Chem. Rev. 2010, 110, 1746-1787.

https://doi.org/10.1021/cr9002424

21. Chang, S.; Grubbs, R. H. J. Org. Chem. 1998, 63, 864-866.

https://doi.org/10.1021/jo9712198

22. Koenig, H.; Goetz, N.; Klein, U.; Eler, K. Patent WO 9703969, 1977; Chem. Abstr. 1997, 126, 199566.

23. Arbačiauskienė, E.; Vilkauskaitè, G.; Eller, G. A.; Holzer, W.; Šačkus, A. Tetrahedron 2009, 65, 7817-7824. https://doi.org/10.1016/j.tet.2009.07.017

24. Li, Y.; Liu, R.; Yan, Z.; Zhang, X.; Zhu, H. Bull. Korean Chem. Soc. 2010, 31, 3341-3347. https://doi.org/10.5012/bkcs.2010.31.11.3341

25. Arbačiauskienė, E.; Laukaitytè, V.; Holzer, W.; Šačkus, A. Eur. J. Org. Chem. 2015, 5663-5670. https://doi.org/10.1002/ejoc.201500541

26. Milišiūnaitè, V.; Arbačiauskienè, E.; Bieliauskas, A.; Vilkauskaitè, G.; Šačkus, A.; Holzer, W. Tetrahedron 2015, 71, 3385-3395.

https://doi.org/10.1016/j.tet.2015.03.092

27. Arbačiauskienė, E.; Kazlauskas, K.; Miasojedovas, A.; Juršėnas, S.; Jankauskas, V.; Holzer, W.; Getautis, V.; Šačkus, A. Synth. Met. 2010, 160, 490-498.

https://doi.org/10.1016/j.synthmet.2009.11.038

28. Arbačiauskienė, E.; Kazlauskas, K.; Miasojedovas, A.; Juršènas, S.; Jankauskas, V.; Holzer, W.; Getautis, V.; Šačkus, A. Dyes Pigm. 2010, 85, 79-85. 
29. Kazlauskas, K.; Kreiza, G.; Arbačiauskienė, E.; Bieliauskas, A.; Getautis, V.; Šačkus, A.; Juršènas, S. J. Phys. Chem. C 2014, 118, 25261-25271.

https://doi.org/10.1021/jp507707f

30. Kazlauskas, K.; Miasojedovas, A.; Dobrovolskas, D.; Arbačiauskienè, E.; Getautis, V.; Šačkus, A.; Juršėnas, S. J. Nanopart. Res. 2012, 14, 877-890. https://doi.org/10.1007/s11051-012-0877-6

31. O’Brien, D. F.; Gates, J. W., Jr. J. Org. Chem. 1966, 31, 1538. https://doi.org/10.1021/jo01343a054

32. Arbačiauskienè, E.; Martynaitis, V.; Krikštolaitytė, S.; Holzer, W.; Šačkus, A. Arkivoc 2011, (xi), 1-21. http://dx.doi.org/10.3998/ark.5550190.0012.b01

33. Maryanoff, B. M.; Reitz, A. B. Chem. Rev. 1989, 89, 863-927. https://doi.org/10.1021/cr00094a007

34. Wittig, G.; Schoellkopf, U. Org. Synth. Coll. 1973, 5, 751.

35. Guedour, H.; Aloui, F.; Beltifa. A.; Mansour, H. B.; Hassine, B. B. C. R. Chimie 2017, 20, 841-849.

36. $A C D / C+H$ Predictors and $D B$, version 10.04; Advanced Chemistry Development, Inc., Toronto, 2006; www.acdlabs.com.

37. Braun, S.; Kalinowski, H.-O.; Berger, S. 150 and More Basic NMR Experiments, 2nd Edn.; Wiley-VCH: New York, 1998.

38. Nyberg, N. T.; Duus, $\varnothing$, J. ; Sørensen, O. W. J. Am. Chem. Soc. 2005, 127, 6154-6155. https://doi.org/10.1021/ja050878w

39. Buevich, A. V. ; Williamson, R. T. ; Martin, G. E. J. Nat. Prod. 2014, 77, 1942-1947. https://doi.org/10.1021/np500445s

40. Yuya, Y.; Sadayuki, A.; Yuichi, M. Pat. Appl. WO 2013162072 A1, 2013.

41. Bueschel, M.; Haremza, S.; Wagenblast, G. Pat. Appl. DE 102005057894 A1, 2007.

42. Li, Y.; Liu, R.; Yan, Z.; Zhang, X.; Zhu, H. Bull. Korean Chem. Soc. 2010, 31, 3341-3347. https://doi.org/10.5012/bkcs.2010.31.11.3341 